\title{
An Overview of Randomization and Minimization Programs for Randomized Clinical Trials
}

\author{
Mahmoud Saghaei \\ Department of Anesthesiology, Medical Image and Signal Processing Research Center, Department of Anesthesia, Isfahan University of Medical \\ Sciences, Isfahan, Iran
}

\section{A B S T R A C T}

Randomization is an essential component of sound clinical trials, which prevents selection biases and helps in blinding the allocations. Randomization is a process by which subsequent subjects are enrolled into trial groups only by chance, which essentially eliminates selection biases. A serious consequence of randomization is severe imbalance among the treatment groups with respect to some prognostic factors, which invalidate the trial results or necessitate complex and usually unreliable secondary analysis to eradicate the source of imbalances. Minimization on the other hand tends to allocate in such a way as to minimize the differences among groups, with respect to prognostic factors. Pure minimization is therefore completely deterministic, that is, one can predict the allocation of the next subject by knowing the factor levels of a previously enrolled subject and having the properties of the next subject. To eliminate the predictability of randomization, it is necessary to include some elements of randomness in the minimization algorithms. In this article brief descriptions of randomization and minimization are presented followed by introducing selected randomization and minimization programs.

Key words: Computer program, minimization, randomization, randomized clinical trial

\section{INTRODUCTION}

As stated in the Consolidated Standards of Reporting Trials, ${ }^{[1]}$ a clear description of the method used to enroll subjects into different arms of a trial is necessary. This, way the reporter declares that no selection bias was present in the trial, and therefore, the trial results are valid. ${ }^{[2]}$ Subjects must be allocated to trial groups randomly. The main purpose of random allocation is to prevent selection biases. In this article different aspects of randomization and minimization are presented, followed by a brief introduction of some common allocation programs.

\section{RANDOMIZATION}

Randomization or random allocation is usually performed by generating a random list of subject allocations prior to the start of a trial. Randomization is usually associated with trial blindness. That is, during randomization each subject will be given a unique identification code (numeral or alpha), which after the completion of the study will be decoded to the actual subject group. Traditional methods of subject allocation to treatment groups, such as, allocating every other subject to each group (alternating allocation) is not considered random allocation. Randomization may be simple or blocked. ${ }^{[3]}$ In simple randomization we construct a randomized list of all the subjects who are going to be enrolled into the trial. Although it is effective to randomize subjects into trial arms, simple randomization may accidentally result in clusters of subjects being given the same treatment in certain periods during the trial. Also it tends to be inefficient with respect to the available resources, which is a problem when the trial involves preparation and blind labeling of drugs prior to the intervention. Block randomization can overcome the problem of subject sequestration and resource inefficiency by dividing the whole set of randomized subjects into smaller blocks. In this manner, after completion of a block, an equal number of subjects will be enrolled in each group. Also the researchers have to prepare and label the drugs for only one block at a time. The main usage of block randomization is for controlling the systematic factors, such as, time and location, which otherwise may affect the outcome of the trial. For example, in multi-center trials, each center may receive a whole block of subjects. Unfortunately, block randomization increases the predictability of subject enrollment near the end of the block boundary, especially when the allocations of already enrolled subjects are known and the blocks have an equal number of subjects from each group. Thus, one may guess the group of the next 
subject, especially if this is the last subject in the block. To overcome this problem we may use variable, but predefined block sizes, with blinding of researchers about the block boundaries. This way the block sizes are different from one another, which reduce the probability of predicting subject membership. Block sizes and numbers can be totally in random, that is the number of blocks and the size of each block can be varied randomly. This is random permuted block randomization.

To allocate subjects to treatment groups randomly, we can use the table of random numbers, tossing a coin, drawing sealed envelopes, and using computer software. Supervising the overall aspects of randomization and keeping records of different allocations, with their labels, is a difficult and errorprone task, which necessitates using advance computer software. Randomization software has the capability to control different aspects of randomization, such as, the type of randomization, generating unique identification codes for each participant and implementation of trial blindness. The typical randomization software has functions and control for selecting the type of randomization from among simple, equal blocks, random fixed permuted blocks, and total permuted blocks. In addition these software offer control over a generation of unique identifier strings to select alpha, numeric or mixed types of unique identifiers in sequential or random orders.

During the last decade many randomization programs have been developed. Some of these programs are downloadable and run on a personal computer (PC), others are used as web services. Although many of these randomization programs are free, a majoritiy of them are not open sources, which prevents close inspection of their algorithm and program functions. In this article some of these softwares are introduced. The selection is based on the availability of service, download, and documentation. This must in no way be considered as an exhaustive list of randomization programs, and the selection is only on availability and ease of use. A comprehensive list of randomization programs is available at the Directory of Randomization Software and Services. ${ }^{[4]}$

\section{Clinstat}

Clinstat is a free, closed source, interactive, multi-module MS-DOS statistical software for carrying out basic statistical analysis, in addition to performing randomization. ${ }^{[5]}$ As it is old, running MS-DOS programs is tricky. Appendix A provides the basic instruction for running an MS-DOS program under the Windows operating system. Upon execution in a DOS console, the program displays a menu for various statistical analyses [Figure 1]. Apart from different statistical modules, there is a randomization module for random number generation, sampling, and allocation of tasks, which can be selected as the eighth

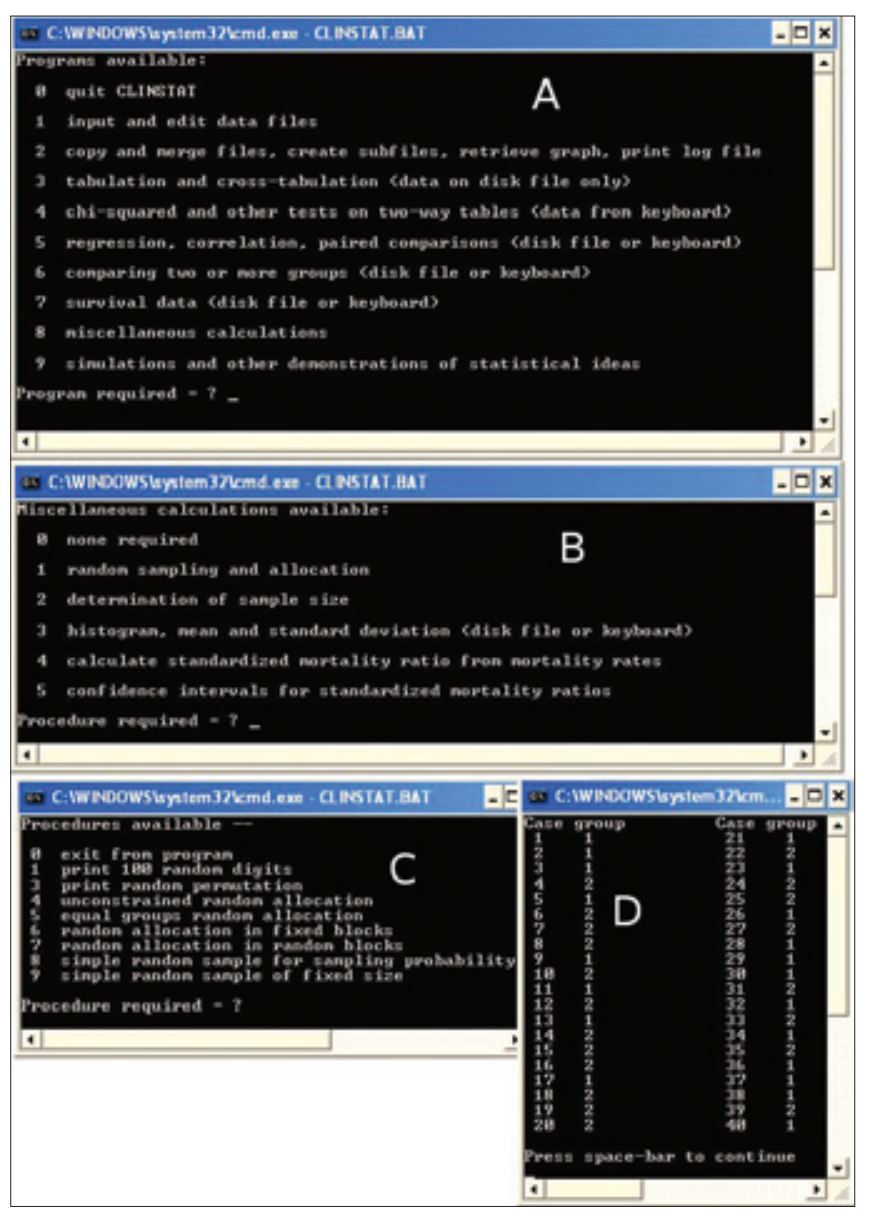

Figure I: Clinstat program. A - The first screen of the Clinstat program showing different statistical utilities. To perform randomization, select option 8 'miscellaneous calculations' from this menu. B - Upon selection of option 8 from the main menu, this menu will be displayed in the Clinstat program. For randomization select 'random sampling and allocation' (option I). C - Different randomization procedures in the Clinstat program. D - An output from the program after selecting 'random allocation in fixed blocks' (menu option 6), showing two blocks of the output

option in the main Clinstat menu (under 'miscellaneous calculations'). In this program the trial groups are unlimited and it randomly allocates up to 1000 subjects into any number of treatment groups. The program support both equal and unequal group sizes. Block randomization (fixed and random sizes) is also supported. Group names or labels cannot be specified, and the subjects are allocated into groups with numeric indicators (that is group 1, 2, 3, ...). Further documentation about the software is available on the program's website.

\section{Randomization.com}

Randomization.com is an online, closed-source, free web service for obtaining randomized lists. ${ }^{[6]}$ This program supports up to 20 groups each, with a unique label. Sequentially each subject is given an ID number, which by default starts from one, but it is possible to specify a different starting point. It consists of three generators. 
The first generator produces random permuted blocks with specified block sizes. Up to four block sizes may be used, and for each block a repeat factor can be specified, which defaults to one [Figure 2]. The second generator is for cross-over studies, where each subject must receive all the treatments in random order. The output determines the order of receiving treatments for each subject. Up to six treatment labels may be entered [Figure 3]. Orders may be produced in random permutations in which some orders may occur more frequently than others, or at balanced permutations, in which different orders occur at the same time. The last generator is for random sampling from a population source. The only thing this generator does is to permute a sequence of integers. In other words it shuffles the sequence in a random way. Separate documentations are available on the service website for each of three generators.

\section{GraphPad QuickCalcs}

GraphPad QuickCalcs is a closed-source, free, online limited randomization service for allocating subjects to a number of groups, with repeated block defaults at one. ${ }^{[7]}$ It can also produce a shuffled list of numbers for each group. No documentation is available for this randomization service.

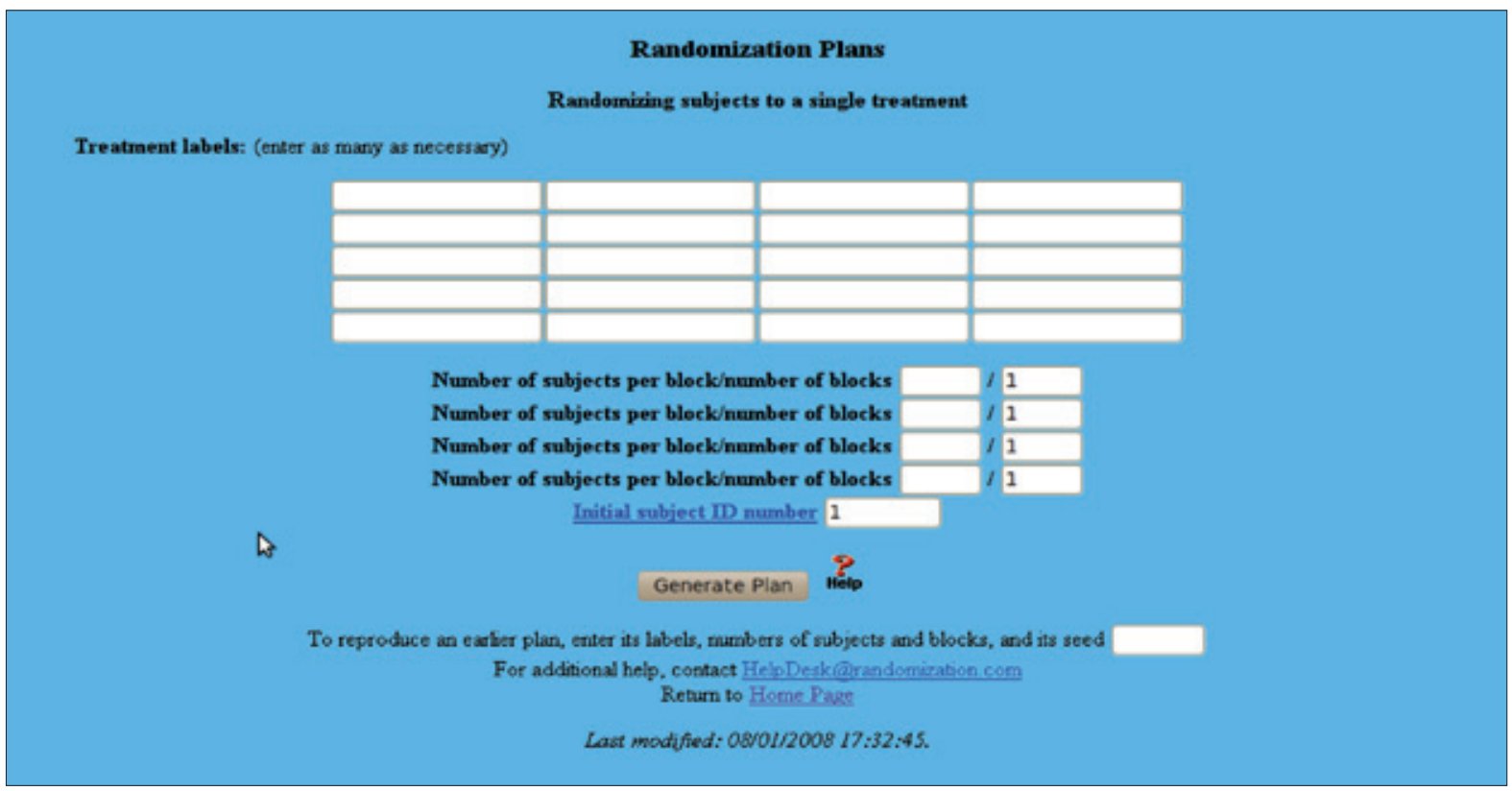

Figure 2: First generator of Randomization.com for random permuted blocks of determined sizes and numbers. Up to 20 group names may be entered. A maximum of four block sizes together with the number of blocks with each size may be specified

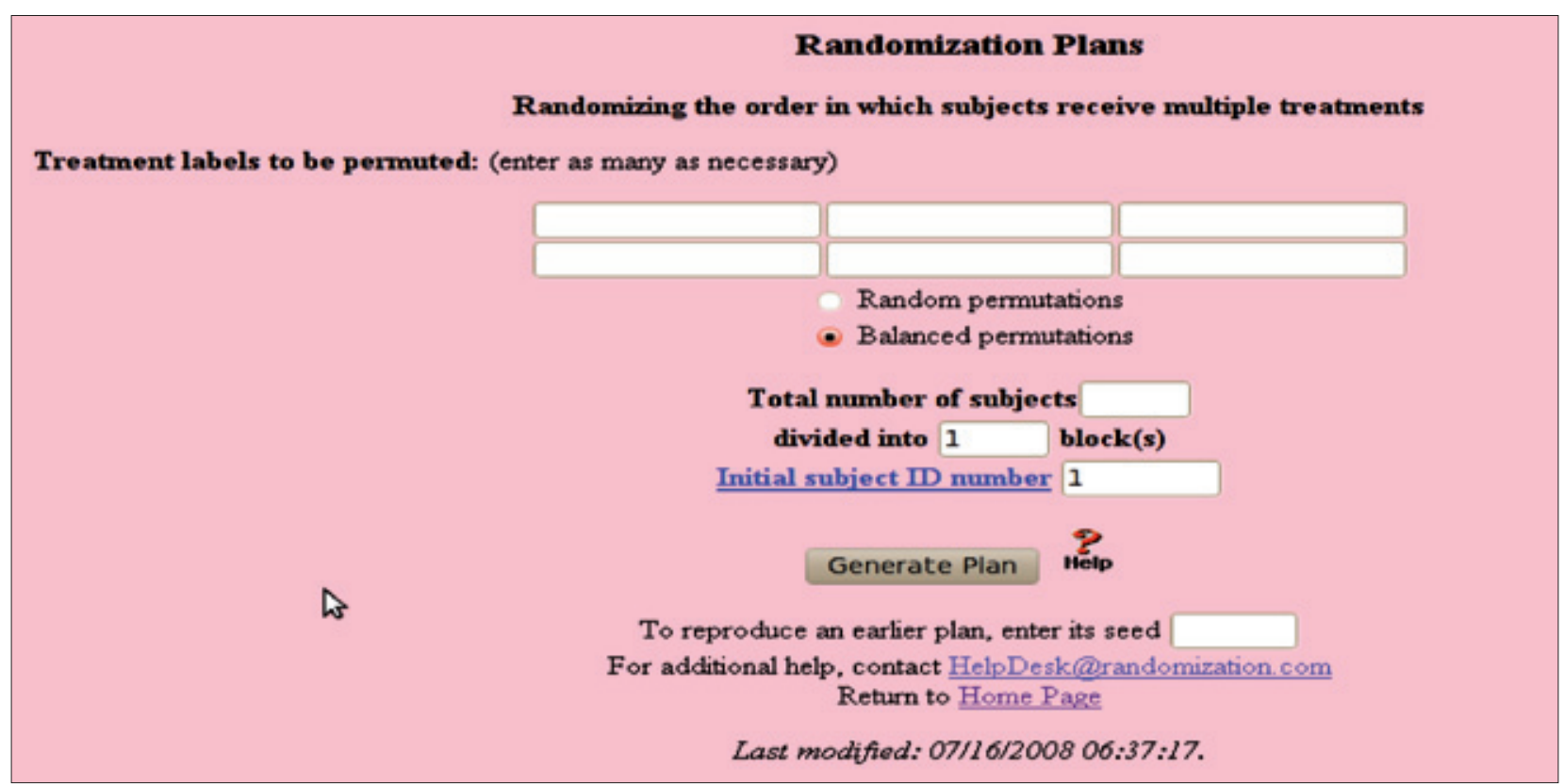

Figure 3: Second generator of Randomization.com for randomizing the order of treatment in cross-over trials. Up to six treatment labels may be specified 


\section{Research Randomizer}

Research Randomizer is also a closed-source, free, randomization web service. ${ }^{[8]}$ It can be used for random sampling, random assignment to treatment groups, and block randomization. It does not support group labels. This randomization service has an excellent tutorial with five sample randomization scenarios for random sampling, random assignment, random block assignment, generating random numbers in a specified range, and random ordering of a set of items.

Random Allocation Software and Service: The Random Allocation Software is a closed, source-free, desktop software, running on the MS-Windows operating system. ${ }^{[9]}$ The Random Allocation Software is also available as a free open source web service. ${ }^{[10,11]}$ Both have different settings for simple and blocked randomizations; length, format, and ordering of generated unique identifiers; type and format of program output; and saving sessions for future use. It permits complete control over the randomization protocol. However, it does not support randomization for cross-over trials or adapted randomization. Complete documentation of the program is published in the BMC Medical Research Methodology. ${ }^{[12]}$

\section{Adaptive Randomization}

This is a closed-source, free desktop software, available for download and running, on the MS-Windows operating system, under the dot net framework. ${ }^{[13]}$ This program supports a specific type of randomization in which the randomization probabilities are affected in favor of those treatments with better outcomes. Two kinds of outcome measures can be fed into the program, which is a binary and time-to-event program. It has a limit of up to ten treatment groups. Also this program supports interim measures and stops rules for terminating a trial when certain criteria are met. It has adaptation factors. The more the adaptation factors, the more will be the probability of their assignment to treatments with better outcomes. The zero of this adaptation factor (no adaptation) means complete randomization. This program is a desktop software, running under the Windows operating system and requires preinstallation of the dot net framework.

\section{MINIMIZATION}

The most important drawback of the randomization software is the problem of unmatched groups. In the process of randomization it is probable that the treatment groups develop significant differences in some prognostic factors, especially when the sample size is relatively small $(<200) .^{[14,15]}$ If these factors have important effects on the primary or secondary outcomes of the study, any important difference in the levels of these factors invalidate the trial results, and necessitate complicated statistical analysis with unreliable results. Various methods have been used to overcome the problem of unmatched trial groups including minimization and stratification, with minimization providing more acceptable results. ${ }^{[16]}$ Taves, ${ }^{[17]}$ in 1974, and Pocock and Simon, ${ }^{[18]}$ in 1975, independently described the method of minimization. With minimization the first subjects are enrolled randomly into one of groups. The subsequent subjects will be allocated to treatment groups after hypothetical allocation of each subject to every group, and then calculating an imbalance score. Using these imbalance scores, we can decide to which group the new subject must be allocated, to have the minimum amount of imbalance, in terms of prognostic factors. Pure minimization is indeed completely deterministic, that is, we can predict which group the next subject will be enrolled in, provided the factor levels of the new subject are known. This may invalidate the principle of trial blindness and introduce some bias into the trial. To overcome this shortcoming some elements of randomness are incorporated into the minimization algorithm, to make the prediction unlikely. Unfortunately the whole process of minimization is well beyond the skill of a typical clinical researcher, especially when the problem of unequal group allocations has to be taken into account. The difficulty in computation has resulted in a relatively less frequent use of minimization methods, in randomized clinical trials. The computer software can perform excellently in these situations, especially when the implementation has been logical. In the following sections, the aspects of two minimization programs are presented. Again the selection of these programs is based on the availability and ease of use.

\section{Minim}

Minim is a free, but closed-source, MS-DOS program, for minimizing subjects into the arms of a clinical trial. ${ }^{[19]}$ This program must be run under the DOS or Windows operation system. Appendix A provides the basic instruction for running an MS-DOS program under the Windows operating system. Minim is an interactive program, which means it prompts for user input, one at a time, then displays the next prompt, and asks for another input. Upon execution it prompts for the data file name, which is the file containing the minimization settings for a trial, together with the already allocated subjects' data if there are any. Therefore, if one has already defined a trial by this program and previously saved it, they can enter its name to load it. Otherwise the name will be used as also the name of the new trial settings. When defining a new trial, the program asks for trial information and the different trial settings. Appendix B is a typical minim program session, which displays the questions the program asks and sample answers to the questions.

To the best of our knowledge this program does not support setting the method and the amount of probability used for 
allocation of subjects to trial groups. Also it does not allow changing the distance measure.

\section{MagMin}

MagMin is an online, closed-source, private minimization service, for blind allocation of subjects to multi-center clinical trials. As this is not a public service, its properties cannot be fully evaluated. However, in an article presenting this program it has been described as a minimization program using the Pocock and Simon's minimization method, ${ }^{[18]}$ using standard deviation as the distance measure. ${ }^{[20]}$ MagMin seems to have limited choice over different aspects of minimization, such as, the choice of distance measure. Anyhow, due to its unavailability it cannot be elucidated for certainty of its full potential. A demo website is available, which shows active allocation. ${ }^{[21]}$ This demo has been previously set up, and in its present form is a running minimization service, which shows allocation of a new subject into the trial without displaying the details of the minimization setting. In the running demo it is possible to add subjects by minimization to an already defined trial. The demo asks for different properties of new subjects and enrolls it into the trial and returns the numerical blind code of the enrolled subject.

\section{MinimPy}

MinimPy is a free, open-source, desktop minimization program, which allocates subjects to treatment groups in a clinical trial. ${ }^{[22]}$ With this program nearly all aspects of a minimization model can be configured. Of special note is the ability to choose distance measure and the probability method. In addition this program supports the biased coin minimization as the probability method, which has not been used in previous programs. MinimPy is produced using the python programming language, ${ }^{[23]}$ which is very strong and efficient for computational purposes, and it is one of the most readable programming languages. MinimPy requires python to be installed, with gtk libraries, for support of graphic user interface. This program has different windows for such things as minimization settings, variables, groups, allocations, and balance [Figure 4]. It also supports pre-loading an already allocated sample into a trial.

\section{CONCLUSIONS}

With the advent of computer program and online services for randomization and minimization in clinical trials, an increasing number of randomized clinical trials are going to make efficient use of them. However, there are few aspects of these program and services that need more attention to make them more acceptable for various needs of clinical researches. Also it can be concluded that there is an increasing need to move toward open-source development, to enhance the quality of the produced software and make them available to the critiques of different clinical and software specialists.

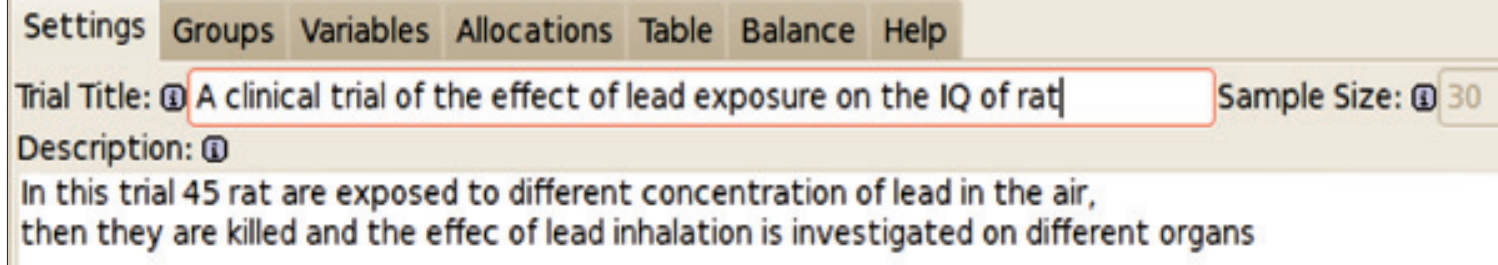

Figure 4: MinimPy program showing different tabs for settings, variables, groups, allocations, table and balance. In this figure the settings tab is displaying variuos aspects of minimization protocol such as probability method and distance measure 


\section{APPENDIX}

\section{Appendix A: Running MS-DOS Programs}

MS-DOS programs usually do not need installation. To run the program you need an MS-DOS operating system. Alternatively, you can run the program in a DOS console under Windows. For the latter, click the start menu and select Run. A dialog window appears. Type 'cmd' (without quotes) in this dialog window, to open a DOS terminal, with the blinking cursor in front of a greater than sign (>). Command and programs are run by typing their names in front of this command prompt. First change the current directory to the one where your program is situated. Here we suppose your program name is prog.exe and it is in the C: \myprog folder:

$$
\begin{aligned}
& >\text { cd } \backslash \\
& >\text { cd myprog } \\
& >\text { prog.exe }
\end{aligned}
$$

\section{Appendix B}

Below is a typical minim program session that displays questions the program asks (in italic) and sample answers to the questions (bold). Texts between braces $(\{\}$.$) are$ comments provided here to further elaborate the answers to questions:

What is the title for this trial? Test Trial

Type variable name No. 1 ("' to end) ? Gender

How many categories has Gender ? 2

What name for category 1 ? Male

What name for category 2? Female

What weighting should Gender have? 1

\{The weight of this variable showing its relative importance. A value of 1 means no special importance

Type variable name no. 2 ("*' to end) ? Age

How many categories has Age? 3

What name for category $1 ?<20 \mathrm{YO}$

What name for category 2 ? $20-50 \mathrm{YO}$

What name for category 3 ? > 50 YO

What weighting should Age have? 1

Type variable name no. 2 ("*' to end) ? *

Give the name of treatment group 1? Acetaminophen

Give a symbol to represent Acetaminophen ? A

Give a value for proportional allocation ? 1

\{The ratio of allocation for this group. It is the partial contribution of this group in total sample size. Default is 1$\}$

Give the name of treatment group 2? Placebo

Give a symbol to represent Placebo ? P

Give a value for proportional allocation ? 1

Give the name of treatment group 3 ( ${ }^{*}$ ' to end) ? *
Test Trial

Acetaminophen Placebo

Gender

Male

0

0

Female

0

0

Age

$<20$ YO

$0 \quad 0$

20-50 YO

0

0

$>50 \mathrm{YO}$

0

0

Total

0

0

0

Is that all correct? Y

Type 'A' for Allocating new patients by minimization, or ' $U$ ' for Updating data with manually entered patients, or ' $S$ ' to StoP $>>A$

Do you have details of a patient ? Y

What is the value of Gender for this patient?

$\begin{array}{ll}\text { Male } & 1 \\ \text { Female } & 2\end{array}$

Value, or code in range 1 to 2 ? 1

What is the value of Age for this patient?

$<20$ YO. 1

$20-50$ YO. 2

$>50$ YO. 3

Value, or code in range 1 to 3 ? 2

Current patient details are:
1. Gender
Male
2. Age
$20-50 \mathrm{YO}$

Do you want to alter any of these details? N

Patient allocated to treatment Placebo.

Do you have details on a patient?

\section{REFERENCES}

1. K. F. Schulz, D. G. Altman, D. Moher, and for the CONSORT Group. CONSORT 2010 Statement: Updated Guidelines for Reporting Parallel Group Randomized Trials. BMC Med 8, pp. 1, 2010.

2. D. J. McEntegart. The pursuit of balance using stratified and dynamic randomization techniques: An overview. Drug Inf J 37, pp. 293-309, 2003.

3. J. L. Fleiss. The Design and Analysis of Clinical Experiments. New York: John Wiley and Sons; 1986.

4. Directory of randomisation software and services. Available from: http://www.users.york.ac.uk/ mb55/guide/randsery.htm.

5. M. Bland. Simple Statistical Software. Available from: http://www.users.york.ac.uk/ $\sim \mathrm{mb} 55 /$ soft/soft.htm.

6. Randomization.com. Available from: http://www.jerrydallal.com/ random/randomize.htm.

7. GraphPad QuickCalcs: Randomly assign subjects to treatment groups. Available from: http:/www.graphpad.com/quickcalcs/randomize1.cfm.

8. Research randomizer: Free random sampling and random assignment Available from: http://www.randomizer.org/index.htm.

9. Random Allocation Software. Available from: http://www.saghaei. net $/ \mathrm{p}=123$.

10. Random Allocation Service. Available from: http://saghaei.net/?page 
$\mathrm{id}=26$.

11. Random Allocation Service Download. Available from: http://saghaei. net/downloads/ra.tar.gz.

12. M. Saghaei. Random Allocation Software. BMC Med Res Methodol Nov 9;4: pp. 26, 2004. Available from: http://www.biomedcentral. com/1471-2288/4/26, [Last accessed on 2004].

13. Adaptive Randomization. https://biostatistics.mdanderson.org/ SoftwareDownload/SingleSoftware.aspx?Software_Id=62. [Last Accessed on 2011 Aug 02].

14. J. M. Lachin. Statistical properties of randomization in clinical trials. Control Clin Trials 9, pp. 289-311, 1988.

15. R. L. Stout, P. W. Wirtz, J. P. Carbonari, and Del Boca FK. Ensuring balanced distribution of prognostic factors in treatment outcome research. J Stud Alcohol Suppl 12, pp. 70-5, 1994.

16. W. N. Kernan, C. M. Viscoli, R. W. Makuch, L. M. Brass, and R. I. Horwitz. Stratified randomization for clinical trials. J Clin Epidemiol 52, pp. 19-26, 1999.

17. D. R. Taves. Minimization: A new method of assigning patients to treatment and control groups. Clin Pharmacol Ther 15, pp. 443-53, 1974.
18. S. J. Pocock, and R. Simon. Sequential treatment assignment with balancing for prognostic factors in the controlled clinical trial. Biometrics 31, pp. 103-15, 1975.

19. Minim: Allocation by minimisation in clinical trials. Available from: http://www.users.york.ac.uk/ mb55/guide/minim.htm.

20. H. W. Cai, J. L. Xia, D. H. Gao, and X. M. Cao. Implementation and experience of a web-based allocation system with Pocock and Simon's minimization methods. Contemp Clin Trials 31, pp. 510-3, 2010.

21. Minimization Random allocation System. Available from: http://www. rct.fmmukq.com/eng (username/password: fmmukq/fmmukq)

22. MinimPy | Download MinimPy software for free at SourceForge.net. Available from: http://sourceforge.net/projects/minimpy

23. Python Programming Language - Official Website. Available from: http://www.python.org. [Last accessed on 2011 Aug 02].

How to cite this article: Saghaei M. An overview of randomization and minimization programs for randomized clinical trials. J Med Sign Sens 2011;1:55-61

Source of Support: Nil, Conflict of Interest: None declared

\section{BIOGRAPHY}

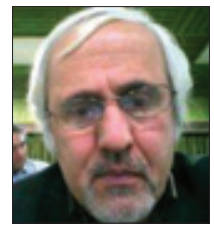

Mahmoud Saghaei is a professor in the Department of Anesthesia at the Faculty of Medicine, Isfahan University of Medical Sciences, Isfahan, Iran. He is also a part time member of Medical Image and Signal Processing Research Center (http://misp. mui.ac.ir/en/), which works on different aspects of biomedical engineering solutions to link the medical society with engineers.

\section{Author Help: Online submission of the manuscripts}

Articles can be submitted online from http://www.journalonweb.com. For online submission, the articles should be prepared in two files (first page file and article file). Images should be submitted separately.

1) First Page File:

Prepare the title page, covering letter, acknowledgement etc. using a word processor program. All information related to your identity should be included here. Use text/rtf/doc/pdf files. Do not zip the files.

2) Article File:

The main text of the article, beginning with the Abstract to References (including tables) should be in this file. Do not include any information (such as acknowledgement, your names in page headers etc.) in this file. Use text/rtf/doc/pdf files. Do not zip the files. Limit the file size to $1 \mathrm{MB}$. Do not incorporate images in the file. If file size is large, graphs can be submitted separately as images, without their being incorporated in the article file. This will reduce the size of the file.

3) Images:

Submit good quality color images. Each image should be less than $4096 \mathrm{~kb}(4 \mathrm{MB})$ in size. The size of the image can be reduced by decreasing the actual height and width of the images (keep up to about 6 inches and up to about $1800 \times 1200$ pixels). JPEG is the most suitable file format. The image quality should be good enough to judge the scientific value of the image. For the purpose of printing, always retain a good quality, high resolution image. This high resolution image should be sent to the editorial office at the time of sending a revised article.

4) Legends:

Legends for the figures/images should be included at the end of the article file. 